\title{
Conformational studies of peptides containing cis-3-hydroxy-D-proline
}

\author{
T. K. Chakraborty,* P. Srinivasu, R. Vengal Rao, S. Kiran Kumar and A. C. Kunwar* \\ Indian Institute of Chemical Technology, Hyderabad 500 007, India \\ E-mail: chakraborty@iict.ap.nic.in
}

\section{Supporting Information}

\section{Contents}

Page No.

1. Experimental procedures

S2

2. VT studies of Peptide 1a: Chemical shift vs Temperature plot

S9

3. VT studies of Peptide 1b: Chemical shift vs Temperature plot

4. ${ }^{1} \mathrm{H}$ NMR spectrum of $\mathbf{1 a}$ in $\mathrm{CDCl}_{3}$

5. NOESY spectrum of $1 \mathrm{a}$ in $\mathrm{CDCl}_{3}$

6. TOCSY spectrum of $\mathbf{1 a}$ in $\mathrm{CDCl}_{3}$ S13

7. ${ }^{1} \mathrm{H}$ NMR spectrum of $\mathbf{1 b}$ in DMSO- $d_{6}$

8. ROESY spectrum of $\mathbf{1 b}$ in DMSO- $d_{6}$

9. TOCSY spectrum of $\mathbf{1 b}$ in DMSO- $d_{6}$ S16

10. ${ }^{13} \mathrm{C}$ NMR spectrum of $\mathbf{4}$ in $\mathrm{CDCl}_{3}$

11. ${ }^{13} \mathrm{C}$ NMR spectrum of 5 in $\mathrm{CDCl}_{3}$

12. ${ }^{13} \mathrm{C}$ NMR spectrum of $\mathbf{1 a}$ in $\mathrm{CDCl}_{3}$ S19

13. ${ }^{13} \mathrm{C}$ NMR spectrum of $\mathbf{1 b}$ in $\mathrm{CDCl}_{3}$ 


\section{Experimental procedures}

General Procedures. All reactions were carried out in oven or flame-dried glassware with magnetic stirring under nitrogen atmosphere using dry, freshly distilled solvents, unless otherwise noted. Reactions were monitored by thin layer chromatography (TLC) carried out on $0.25 \mathrm{~mm}$ silica gel plates with UV light, $\mathrm{I}_{2}, 7 \%$ ethanolic phosphomolybdic acid-heat and $2.5 \%$ ethanolic anisaldehyde (with $1 \% \mathrm{AcOH}$ and $3.3 \%$ conc. $\mathrm{H}_{2} \mathrm{SO}_{4}$ )-heat as developing agents. Silica gel finer than 200 mesh was used for flash column chromatography. Yields refer to chromatographically and spectroscopically homogeneous materials unless otherwise stated. Melting points are uncorrected. IR spectra were recorded as neat liquids or $\mathrm{KBr}$ pellets on FT-IR. Mass spectra were obtained under liquid secondary ion mass spectrometric (LSIMS) technique. Optical rotations were measured in $\mathrm{CHCl}_{3}$ solutions with a digital polarimeter.

Details of NMR studies. NMR spectra of the peptides $\mathbf{1 a}, \mathbf{b}$ were recorded in $500 \mathrm{MHz}$ spectrometer at $30{ }^{\circ} \mathrm{C}$ with 3-6 mM solutions in appropriate solvents using TMS as internal standard or the solvent signals as secondary standards and the chemical shifts are shown in $\delta$ scales. Multiplicities of NMR signals are designated as s (singlet), d (doublet), t (triplet), $\mathrm{q}$ (quartet), br (broad), $\mathrm{m}$ (multiplet, for unresolved lines), etc. ${ }^{13} \mathrm{C}$ NMR spectra were recorded at 50 and $75 \mathrm{MHz}$ with complete proton decoupling. The chemical shift assignments were carried out with the help of two-dimensional total correlation spectroscopy (TOCSY) ${ }^{1}$ and nuclear Overhauser effect spectroscopy (NOESY)/rotating frame nuclear Overhauser effect spectroscopy (ROESY) experiments, ${ }^{2}$ the later also provided the information on the proximity of protons. All the experiments were carried out in the phase sensitive mode. ${ }^{3}$ The spectra were acquired with 2 $\times 256$ or $2 \times 192$ free induction decays (FID) containing 8-16 transients with relaxation delays of 1.0 to $1.5 \mathrm{sec}$. The ROESY experiments were performed with mixing time of $0.3 \mathrm{sec}$. For ROESY experiments a spin-locking field of about $2 \mathrm{kHz}$ was used. The TOCSY experiments were performed with the spin locking fields of about $10 \mathrm{kHz}$ and a mixing time of $0.08 \mathrm{sec}$. The two-dimensional data were processed with Gaussian apodization in both the dimensions. To obtain the temperature coefficients of NH-chemical shifts, the spectra were recorded between 30 and $70{ }^{\circ} \mathrm{C}$ (at $30,40,50,60$, and $70{ }^{\circ} \mathrm{C}$ ) in DMSO- $d_{6}$. The temperature coefficients $\Delta \delta / \Delta T$ were determined from the slopes of the linear regression lines obtained from the chemical shift vs temperature plots. ${ }^{4}$ 
Details of Molecular dynamics syudies. Molecular mechanics/dynamics calculations were carried out using Sybyl 6.8 program on a Silicon Graphics O2 workstation. The Tripos force field, with default parameters, was used throughout the simulations.

A dielectric constant of 47 Debye for DMSO solvent was used in all minimizations as well as in MD runs. Minimizations were done first with steepest decent, followed by conjugate gradient methods for a maximum of 2000 iterations each or RMS deviation of $0.005 \mathrm{kcal} / \mathrm{mol}$, whichever was earlier. The energy-minimized structures were then subjected to MD studies. A number of inter atomic distance constraints (more than three bond away) were used in the MD studies that were derived from the rOe cross-peaks from the ROESY spectra in DMSO- $d_{6}$. Distance constraints with a force constant of $15 \mathrm{kcal} / \AA$ were applied in the form of flat bottom potential well with a common lower bound of $2.0 \AA$ and the upper bound of $2.8,3.5$, and $4.0 \AA$, in accordance with the nOe intensities for strong, medium and weak, respectively. ${ }^{5}$ For all the amide bonds, the torsional angle $180^{\circ}$ was used as a constraint. A force constant of $5 \mathrm{kcal} / \AA$ was employed for the dihedral angle constraints. ${ }^{5}$ No H-bonding constraint was used. The energyminimized structures were subjected to constrained MD simulations for duration of $600 \mathrm{ps}$ using 100 cycles, each of 6 ps period, of the Simulated Annealing protocol. The atomic velocities were applied following Boltzmann distribution about the center of mass, to obtain a starting temperature of $700 \mathrm{deg} \mathrm{K}^{6}$

After simulating for $1 \mathrm{ps}$ at high temperature, the system temperature was reduced exponentially over a 5 ps period to reach a final temperature of $300^{\circ} \mathrm{K}$. Structures were sampled after every five cycles, leading to an ensemble of total 20 structures. The sampled structures were energy-minimized without constraints, by using the above-mentioned protocol and the superimposed structures obtained by backbone alignment are shown in the paper in Figures 2 and 4. To determine the backbone and the average pair-wise heavy atom RMSD, the structures were analyzed using the MOLMOL program. ${ }^{7}$

Synthesis of compound 4. To a stirred solution of $\mathbf{3}(1.28 \mathrm{~g}, 2.81 \mathrm{mmol})$ in dry THF (15 $\mathrm{mL})$ was added TBAF ( $1 \mathrm{M}$ in THF, $4.2 \mathrm{~mL}, 4.2 \mathrm{mmol})$ at $0{ }^{\circ} \mathrm{C}$. The reaction mixture was stirred at room temperature for $4 \mathrm{~h}$, quenched with saturated $\mathrm{NH}_{4} \mathrm{Cl}$ solution, and extracted with EtOAc. The combined organic extracts were washed with water, brine, dried $\left(\mathrm{Na}_{2} \mathrm{SO}_{4}\right)$, and concentrated in vacuo. Purification by column chromatography $\left(\mathrm{SiO}_{2}, 70-80 \%\right.$ EtOAc in petroleum ether eluant) afforded the pure product $4(580 \mathrm{mg}, 95 \%)$ as a liquid. $R_{f}=0.3$ (silica gel, $80 \%$ EtOAc in petroleum ether); $[\alpha]^{23} 55.7\left(c 0.63, \mathrm{CHCl}_{3}\right)$; IR (neat) $v_{\max } 3388,2925,1662,1403,1160 \mathrm{~cm}^{-1}$; 
${ }^{1} \mathrm{H}$ NMR $\left(\mathrm{CDCl}_{3}, 400 \mathrm{MHz}\right) \delta$ 4.58-4.46 (m, $\left.1 \mathrm{H}\right)$, 4.04-3.96 (m, $\left.1 \mathrm{H}\right), 3.92-3.81$ (m, $\left.2 \mathrm{H}\right)$, 3.53$3.42(\mathrm{~m}, 2 \mathrm{H}), 2.64$ (broad, $1 \mathrm{H}), 2.10-1.98(\mathrm{~m}, 1 \mathrm{H}), 1.96-1.87(\mathrm{~m}, 1 \mathrm{H}), 1.47$ (s, $9 \mathrm{H}, \mathrm{Boc}) ;{ }^{13} \mathrm{C}$ $\mathrm{NMR}\left(\mathrm{CDCl}_{3}, 75 \mathrm{MHz}\right) \delta 156.0,80.1,72.4,62.1,44.6,32.5,29.6,28.4 ;$ MS (LSIMS) $m / z(\%)$ $218(10)[\mathrm{M}+\mathrm{H}]^{+}$.

Synthesis of compound 5. Diol $4(513 \mathrm{mg}, 2.36 \mathrm{mmol})$ in $\mathrm{CH}_{2} \mathrm{Cl}_{2}(10 \mathrm{~mL})$ was treated with $\mathrm{Et}_{3} \mathrm{~N}(0.49 \mathrm{~mL}, 3.54 \mathrm{mmol})$ at $0{ }^{\circ} \mathrm{C}$. After $5 \mathrm{~min}, \mathrm{TrCl}(724 \mathrm{mg}, 2.60 \mathrm{mmol})$ followed by DMAP (28 mg, $0.236 \mathrm{mmol}$ ) were added and stirred at room temperature for $12 \mathrm{~h}$. It was then diluted with EtOAc, washed with saturated $\mathrm{NH}_{4} \mathrm{Cl}$ solution, water, brine, dried $\left(\mathrm{Na}_{2} \mathrm{SO}_{4}\right)$, and concentrated in vacuo. Purification by column chromatography $\left(\mathrm{SiO}_{2}, 25-30 \%\right.$ EtOAc in petroleum ether eluant) afforded the pure product, which was used in the next step without characterization.

To a solution of tritylated product $(2.36 \mathrm{mmol})$ in THF $(10 \mathrm{~mL}), \mathrm{NaH}(60 \%$ dispersion in oil, $141 \mathrm{mg}, 3.54 \mathrm{mmol}$ ) was added under nitrogen atmosphere at $0{ }^{\circ} \mathrm{C}$. After the mixture was stirred for $10 \mathrm{~min}$ at the same temperature, $\mathrm{BnBr}(0.33 \mathrm{~mL}, 2.83 \mathrm{mmol})$ followed by TBAI $(87$ $\mathrm{mg}, 0.236 \mathrm{mmol}$ ) were added, temperature was slowly raised from $0{ }^{\circ} \mathrm{C}$ to room temperature and stirring was continued for $8 \mathrm{~h}$. The mixture was then diluted with EtOAc, washed with saturated $\mathrm{NH}_{4} \mathrm{Cl}$ solution, water, brine, and dried $\left(\mathrm{Na}_{2} \mathrm{SO}_{4}\right)$. It was then concentrated in vacuo and purification by column chromatography $\left(\mathrm{SiO}_{2}, 12-14 \%\right.$ EtOAc in petroleum ether eluant) afforded the pure product, which was used in the next step without characterization.

To an ice-cold solution of the benzyl protected compound (2.36 mmol), obtained above and dissolved in dry ether $(30 \mathrm{~mL})$, formic acid $(30 \mathrm{~mL})$ was added and stirred for $10 \mathrm{~min}$. Then the reaction mixture was diluted with EtOAc, carefully quenched with solid $\mathrm{NaHCO}_{3}$ until there was no effervescence. The organic layer was washed with water, brine, dried $\left(\mathrm{Na}_{2} \mathrm{SO}_{4}\right)$, and concentrated in vacuo. The residue was purified by column chromatography $\left(\mathrm{SiO}_{2}, 22-24 \%\right.$ EtOAc in petroleum ether eluant) to give pure product 5 (530 mg, $73 \%$ from 4). $R_{f}=0.4$ (silica gel, $40 \%$ EtOAc in petroleum ether); $[\alpha]^{26} 54.5$ (c 0.84, $\mathrm{CHCl}_{3}$ ); IR (neat) $v_{\max } 3403,2925$, 1662, 1388, $1152 \mathrm{~cm}^{-1} ;{ }^{1} \mathrm{H} \mathrm{NMR}\left(\mathrm{CDCl}_{3}, 200 \mathrm{MHz}\right) \delta$ 7.38-7.30 (m, $\left.5 \mathrm{H}, \mathrm{ArH}\right), 4.68-4.45(\mathrm{ABq}$, $\left.2 \mathrm{H}, \mathrm{PhCH}_{2} \mathrm{O}\right), 4.21-4.11(\mathrm{~m}, 1 \mathrm{H}), 4.05-3.83(\mathrm{~m}, 2 \mathrm{H}), 3.79(\mathrm{dd}, J=11.3,7.5 \mathrm{~Hz}, 1 \mathrm{H}), 3.50-$ $3.32(\mathrm{~m}, 2 \mathrm{H}), 2.08-1.89(\mathrm{~m}, 2 \mathrm{H}), 1.46$ (s, $9 \mathrm{H}, \mathrm{Boc}) ;{ }^{13} \mathrm{C} \mathrm{NMR}\left(\mathrm{CDCl}_{3}, 50 \mathrm{MHz}\right) \delta 156.1$, $137.5,128.4,127.8,127.5,80.0,78.9,71.8,63.1,61.9,44.4,29.2,28.4 ;$ MS (LSIMS) $m / z(\%)$ 
208 (46) $\left[\mathrm{M}+\mathrm{H}-\mathrm{C}_{5} \mathrm{H}_{8} \mathrm{O}_{2}\right]^{+}, 308$ (50) $[\mathrm{M}+\mathrm{H}]^{+}$; HRMS (LSIMS): calcd for $\mathrm{C}_{17} \mathrm{H}_{26} \mathrm{NO}_{4}[\mathrm{M}+$ $\mathrm{H}]^{+}$308.1861, found 308.1862.

Synthesis of Compound 6. To a solution of 5 (300 mg, $0.977 \mathrm{mmol})$ in dry DMF (6 $\mathrm{mL})$, PDC $(1.83 \mathrm{~g}, 4.88 \mathrm{mmol})$ was added at $0{ }^{\circ} \mathrm{C}$. The reaction mixture was stirred at room temperature for $24 \mathrm{~h}$ under nitrogen atmosphere. It was then diluted with EtOAc, washed with saturated $\mathrm{CuSO}_{4}$ solution, water, brine, and dried $\left(\mathrm{Na}_{2} \mathrm{SO}_{4}\right)$. It was then concentrated in vacuo to get the acid.

To a solution of Boc-Gly-Leu-NHMe $(352 \mathrm{mg}, 1.17 \mathrm{mmol})$ in dry $\mathrm{CH}_{2} \mathrm{Cl}_{2}(3 \mathrm{~mL})$, trifluoroacetic acid $(1 \mathrm{~mL})$ was added at $0{ }^{\circ} \mathrm{C}$ and the reaction mixture was stirred for $1 \mathrm{~h}$ at room temperature. It was then concentrated in vacuo to give TFA. $\mathrm{H}_{2} \mathrm{~N}-\mathrm{Gly}-\mathrm{Leu}-\mathrm{NHMe}$.

In another round bottom flask, the crude acid $(0.977 \mathrm{mmol})$, prepared above and dissolved in dry $\mathrm{CH}_{2} \mathrm{Cl}_{2}(3 \mathrm{~mL})$, was sequentially treated with $\mathrm{HOBt} . \mathrm{H}_{2} \mathrm{O}$ (158 mg, $\left.1.17 \mathrm{mmol}\right)$ and EDCI (224 mg, $1.17 \mathrm{mmol})$ at $0{ }^{\circ} \mathrm{C}$. After $15 \mathrm{~min}$, TFA. $\mathrm{H}_{2} \mathrm{~N}-\mathrm{Gly}-\mathrm{Leu}-\mathrm{NHMe}$, prepared above and dissolved in $\mathrm{CH}_{2} \mathrm{Cl}_{2}$-DMF $(3: 1,4 \mathrm{~mL})$ was added to the reaction mixture followed by DIPEA $(0.33 \mathrm{~mL}, 1.95 \mathrm{mmol})$. After being stirred for $12 \mathrm{~h}$ at room temperature, the reaction mixture was diluted with EtOAc, washed with saturated $\mathrm{NH}_{4} \mathrm{Cl}$ solution, saturated $\mathrm{NaHCO}_{3}$ solution, water, brine, dried $\left(\mathrm{Na}_{2} \mathrm{SO}_{4}\right)$, and concentrated in vacuo. Purification by column chromatography $\left(\mathrm{SiO}_{2}, 5-6 \% \mathrm{MeOH}\right.$ in $\mathrm{CHCl}_{3}$ eluant) afforded pure peptide 6 (365 mg, 74\%). $R_{f}$ $=0.3$ (silica gel, 6\% $\mathrm{MeOH}$ in $\left.\mathrm{CHCl}_{3}\right) ;[\alpha]^{23}{ }_{\mathrm{D}}-2.6\left(c 0.7, \mathrm{CHCl}_{3}\right) ; \mathrm{IR}(\mathrm{KBr}) v_{\max } 3294,1662$, 1529, $1396 \mathrm{~cm}^{-1}$; ${ }^{1} \mathrm{H}$ NMR $\left(\mathrm{CDCl}_{3}, 300 \mathrm{MHz}\right) \delta$ 7.37-7.26 (m, $6 \mathrm{H}, \mathrm{Ar} H$ and $\left.\mathrm{NH}\right), 6.51(\mathrm{~d}, J=$ $4.5 \mathrm{~Hz}, 1 \mathrm{H}), 6.45-6.34(\mathrm{~m}, 1 \mathrm{H}), 4.61\left(\mathrm{ABq}, J=12.0 \mathrm{~Hz}, 2 \mathrm{H}, \mathrm{PhCH}_{2} \mathrm{O}\right), 4.38(\mathrm{~d}, J=6.0 \mathrm{~Hz}, 1$ H), 4.35 (t, $J=5.2 \mathrm{~Hz}, 1 \mathrm{H}), 4.29$ (d, $J=6.0 \mathrm{~Hz}, 1 \mathrm{H}), 4.09(\mathrm{~m}, 1 \mathrm{H}), 3.78$ (m, $1 \mathrm{H}), 3.65-3.48$ (m, 2 H), 2.74 (d, J = 4.5 Hz, 3 H, NMe), 2.21-2.06 (m, 1 H), 2.05-1.92 (m, 1 H), 1.76-1.64 (m, 3 H), 1.43 (s, 9 H, Boc), 0.90 ( d, $J=7.5 \mathrm{~Hz}, 3 \mathrm{H}$, Leu $\delta H$ ), 0.87 (d, $J=6.0 \mathrm{~Hz}, 3 \mathrm{H}, \mathrm{Leu} \delta H^{\prime}$ ); MS (LSIMS) $m / z(\%) 405(40)\left[\mathrm{M}+\mathrm{H}-\mathrm{C}_{5} \mathrm{H}_{8} \mathrm{O}_{2}\right]^{+}, 505$ (14) $[\mathrm{M}+\mathrm{H}]^{+}$; HRMS (LSIMS): calcd for $\mathrm{C}_{21} \mathrm{H}_{33} \mathrm{~N}_{4} \mathrm{O}_{4}\left[\mathrm{M}+\mathrm{H}-\mathrm{C}_{5} \mathrm{H}_{8} \mathrm{O}_{2}\right]^{+}$405.2502, found 405.2509.

Synthesis of peptide 1a. To a solution of 6 (200 mg, $0.396 \mathrm{mmol})$ in dry $\mathrm{CH}_{2} \mathrm{Cl}_{2}(3 \mathrm{~mL})$, trifluoroacetic acid $(1 \mathrm{~mL})$ was added at $0{ }^{\circ} \mathrm{C}$ and stirred under nitrogen for $1 \mathrm{~h}$ at room temperature. The reaction mixture was then concentrated in vacuo to give TFA.D-cis-3Hyp(Bn)-Gly-Leu-NHMe. 
In another round bottom flask, Boc-Val-OH (103 mg, $0.476 \mathrm{mmol})$, dissolved in dry $\mathrm{CH}_{2} \mathrm{Cl}_{2}(3 \mathrm{~mL})$, was sequentially treated with $\mathrm{HOBt} . \mathrm{H}_{2} \mathrm{O}(64 \mathrm{mg}, 0.476 \mathrm{mmol})$ and EDCI (91 $\mathrm{mg}, 0.476 \mathrm{mmol})$ at $0{ }^{\circ} \mathrm{C}$. After $15 \mathrm{~min}$, TFA.D-cis-3-Hyp(Bn)-Gly-Leu-NHMe, prepared above and dissolved in dry $\mathrm{CH}_{2} \mathrm{Cl}_{2}(4 \mathrm{~mL})$ was added to the reaction mixture followed by DIPEA $(0.13$ $\mathrm{mL}, 0.793 \mathrm{mmol})$. After stirring for $12 \mathrm{~h}$ at room temperature, the reaction mixture was diluted with EtOAc, washed with saturated $\mathrm{NH}_{4} \mathrm{Cl}$ solution, saturated $\mathrm{NaHCO}_{3}$ solution, water, brine, dried $\left(\mathrm{Na}_{2} \mathrm{SO}_{4}\right)$, and concentrated in vacuo. Purification by column chromatography $\left(\mathrm{SiO}_{2}, 4-5 \%\right.$ $\mathrm{MeOH}$ in $\mathrm{CHCl}_{3}$ eluant) gave pure peptide $1 \mathrm{a}\left(204 \mathrm{mg}, 85 \%\right.$ ). $R_{f}=0.32$ (silica gel, 6\% $\mathrm{MeOH}$ in $\left.\mathrm{CHCl}_{3}\right) ;[\alpha]^{26}-29.2\left(c 1.05, \mathrm{CHCl}_{3}\right) ; \operatorname{mp} 85-88^{\circ} \mathrm{C}$; IR (KBr) $v_{\max } 3301,2941,1662,1505,1152$ $\mathrm{cm}^{-1} ;{ }^{13} \mathrm{C} \mathrm{NMR}\left(\mathrm{CDCl}_{3}, 75 \mathrm{MHz}\right) \delta 172.9,172.4,169.3,168.5,156.5,137.5,128.4,127.8$, $127.4,80.4$, 72.3, 65.4, 58.1, 52.2, 45.7, 43.2, 40.3, 31.0, 29.8, 29.6, 28.2, 26.1, 24.6, 23.1, 21.5, 19.5, 18.4; MS (LSIMS) $m / z(\%) 504$ (42) $\left[\mathrm{M}+\mathrm{H}-\mathrm{C}_{5} \mathrm{H}_{8} \mathrm{O}_{2}\right]^{+}, 604(38)[\mathrm{M}+\mathrm{H}]^{+}$.

Synthesis of peptide $1 \mathbf{b}$. To a solution of $1 \mathrm{a}(100 \mathrm{mg}, 0.165 \mathrm{mmol})$ in $\mathrm{MeOH}(4 \mathrm{~mL}), \mathrm{Pd}(\mathrm{OH})_{2^{-}}$ C (20\%, $50 \mathrm{mg})$ was added and the mixture was hydrogenated under atmospheric pressure using a $\mathrm{H}_{2}$-filled balloon for $2 \mathrm{~h}$. It was then filtered through a short pad of Celite and the filter cake was washed with $\mathrm{MeOH}$. The combined filtrate and washings were concentrated in vacuo. Purification by column chromatography $\left(\mathrm{SiO}_{2}, 7-8 \% \mathrm{MeOH}\right.$ in $\mathrm{CHCl}_{3}$ eluant) gave pure peptide $1 \mathbf{b}(80 \mathrm{mg}, 94 \%) . R_{f}=0.5$ (silica gel, $15 \% \mathrm{MeOH}$ in $\left.\mathrm{CHCl}_{3}\right) ;[\alpha]^{23} \mathrm{D} 3.6\left(c 1.3, \mathrm{CHCl}_{3}\right)$; mp $103-$ $105{ }^{\circ} \mathrm{C}$; IR (KBr) $v_{\max } 3317,2956,2909,1654,1537,1152 \mathrm{~cm}^{-1} ;{ }^{13} \mathrm{C} \mathrm{NMR}\left(\mathrm{CDCl}_{3}, 75 \mathrm{MHz}\right) \delta$ 173.3, 172.1, 170.0, 169.6, 156.5, 80.2, 70.8, 65.3, 57.7, 51.9, 45.5, 43.3, 40.5, 33.4, 30.2, 29.6,

29.3, 28.3, 26.2, 24.6, 23.0, 21.6, 19.4, 18.2; MS (LSIMS) $m / z(\%) 414$ (62) $\left[\mathrm{M}+\mathrm{H}-\mathrm{C}_{5} \mathrm{H}_{8} \mathrm{O}_{2}\right]^{+}$, $514(66)[\mathrm{M}+\mathrm{H}]^{+}$.

\section{References}

1. (a) Cavanagh, J.; Fairbrother, W. J.; Palmer III, A. G.; Skelton, N. J. Protein NMR Spectroscopy, Academic Press: San Diego, 1996. (b) Wüthrich, K. NMR of Proteins and Nucleic Acids; Wiley: New York, 1986.

2. Hwang, T. L.; Shaka, A. J. J. Am. Chem. Soc. 1992, 114, 3157-3159.

3. States, D. J.; Haberkorn, R. A.; Ruben, D. J. J. Magn. Reson. 1982, 48, 286-292.

4. (a) Adams, P. D.; Chen, Y.; Ma, K.; Zagorski, M. G.; Sönnichsen, F. D.; McLaughlin, M. L.; Barkley, M. D. J. Am. Chem. Soc. 2002, 124, 9278-9286. (b) Kessler, H.; Bats, J. W.; 
Griesinger, C.; Koll, S.; Will, M.; Wagner, K. J. Am. Chem. Soc. 1988, 110, 1033-1049. (c) Kessler, H. Angew. Chem. Int. Ed. Engl. 1982, 21, 512-523.

5. Kessler, H.; Griesinger, C.; Lautz, J.; Müller, A.; F. van Gunsteren, W.; Berendsen, H. J. C. J. Am. Chem. Soc. 1988, 110, 3393-3396.

6. For details see: (a) Sybyl Force Field Manual, version 6.6, 1999, pp 217-229 (http://www.tripos.com/custResources/applicationNotes/triad/index.html). (b) Evans, J. N. S. Biomolecular NMR Spectroscopy, Oxford University Press: Oxford, 1995.

7. Etezady-Esfarjani, T.; Hilty, C.; Wüthrich, K; Rueping, M.; Schreiber, J; Seebach, D. Helv. Chim. Acta 2002, 85, 1197-1209.

NOE constraints used in MD simulation study.

\begin{tabular}{|c|c|c|c|c|c|c|c|}
\hline \multicolumn{4}{|c|}{ Peptide 1a } & \multicolumn{4}{|c|}{ Peptide 1b } \\
\hline S1. I & From & To & Intensity & Sl No. & From & To & Intensity \\
\hline 1. & GlyNH & LeuNH & $\mathrm{m}$ & 1. & ValC $\alpha \mathrm{H}$ & НypC $\delta \mathrm{H}$ & $\mathrm{S}$ \\
\hline 2. & LeuNH & NHMe & $\mathrm{m}$ & 2. & $\mathrm{ValC} \alpha \mathrm{H}$ & НypC $\delta \mathrm{H}^{\prime}$ & S \\
\hline 3. & LeuNH & GlyC $\alpha \mathrm{H}$ & $\mathrm{S}$ & 3. & LeuNH & NHMe & $\mathrm{m}$ \\
\hline 4. & LeuNH & GlyC $\alpha \mathrm{H}^{\prime}$ & $\mathrm{S}$ & 4. & LeuNH & GlyNH & $\mathrm{m}$ \\
\hline 5. & GlyNH & НурС $\alpha \mathrm{H}$ & $\mathrm{m}$ & 5. & LeuNH & НурС $\alpha \mathrm{H}$ & W \\
\hline 6. & GlyNH & НурС $\beta \mathrm{H}$ & $\mathrm{W}$ & 6. & LeuNH & НурОН & $\mathrm{W}$ \\
\hline 7. & GlyNH & НypC $\delta \mathrm{H}$ & $\mathrm{W}$ & 7. & LeuNH & НурС $\beta \mathrm{H}$ & $\mathrm{W}$ \\
\hline 8. & $\mathrm{ValC} \alpha \mathrm{H}$ & НурС $\delta \mathrm{H}$ & S & 8. & GlyNH & НурОН & $\mathrm{m}$ \\
\hline 9. & $\mathrm{ValC} \alpha \mathrm{H}$ & НypC $\delta \mathrm{H}^{\prime}$ & S & 9. & GlyNH & НурС $\beta \mathrm{H}$ & $\mathrm{m}$ \\
\hline 10. & $\mathrm{ValC} \gamma \mathrm{Me}$ & НурС $\delta \mathrm{H}$ & $\mathrm{m}$ & 10. & LeuNH & GlyC $\alpha \mathrm{H}$ & S \\
\hline 11. & ValC $\gamma \mathrm{Me}$ & НурС $\delta \mathrm{H}^{\prime}$ & $\mathrm{m}$ & 11. & NHMe & LeuC $\alpha \mathrm{H}$ & $\mathrm{S}$ \\
\hline 12. & LeuNH & НурС $\alpha \mathrm{H}$ & $\mathrm{W}$ & 12. & GlyNH & НурС $\alpha \mathrm{H}$ & $\mathrm{S}$ \\
\hline 13. & LeuNH & ValNH & $\mathrm{W}$ & 13. & НурС $\alpha \mathrm{H}$ & НурС $\delta \mathrm{H}$ & W \\
\hline 14. & ValNH & NHMe & W & & & & \\
\hline 15. & BocMe & NHMe & $\mathrm{m}$ & & & & \\
\hline
\end{tabular}


16. NHMe

LeuC $\alpha \mathrm{H}$

S

$\underline{\text { Statistics of Peptide 1a \& } 1 b \text { (averages of } 20 \text { structures) }}$

Peptide 1a

Bond stretching energy

Angle bending energy

Torsional energy

Out of plane bending energy

1-4 van der Waal energy

Van der Waal energy

1-4 electrostatic energy

Electrostatic energy

Fixed torsion energy

Fixed distance range energy

Total energy
$2.079 \pm 0.13$

$13.137 \pm 0.82$

$10.880 \pm 0.95$

$0.096 \pm 0.05$

$5.377 \pm 0.22$

$-15.824 \pm 1.31$

$19.525 \pm 0.89$

$-21.101 \pm 1.38$

$0.002 \pm 0.001$

$0.022 \pm 0.02$

$14.195+1.92$
Peptide 1b

$1.957 \pm 0.11$

$11.985 \pm 0.96$

$9.327 \pm 0.67$

$0.054 \pm 0.03$

$4.504 \pm 0.36$

$-10.356 \pm 1.53$

$18.177 \pm 1.04$

$-18.494 \pm 1.43$

$0.002 \pm 0.001$

$0.026 \pm 0.04$

$17.179+2.39$ 
Chemical shift vs Temperature plot for the amide protons in the ${ }^{1}$ H NMR VT studies of Peptide 1a

Chemical shifts of amide protons of peptide $1 \mathrm{a}$ at various temperatures

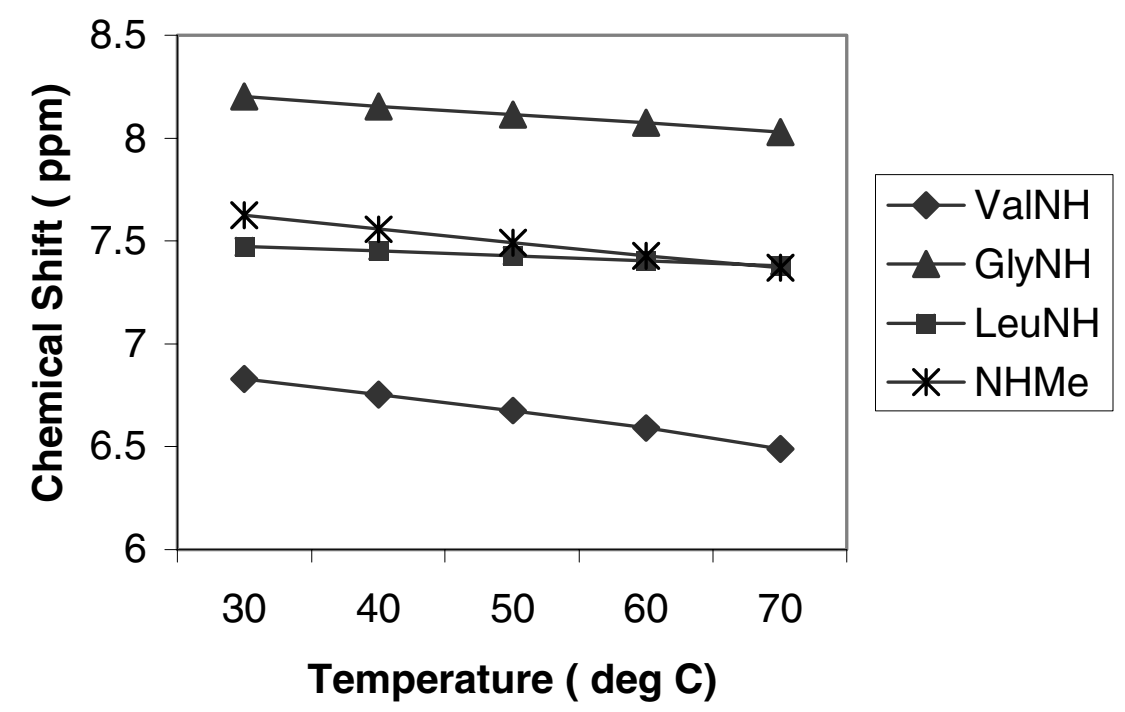

Amide Proton Chemical Shifts obtained during the Variable Temperature Experiment of Peptide 1a

\begin{tabular}{|c|c|c|c|c|}
\hline & Val & Gly & Leu & NHMe \\
\hline $30^{\circ} \mathrm{C}$ & 6.830 & 8.204 & 7.473 & 7.628 \\
\hline $40{ }^{\circ} \mathrm{C}$ & 6.753 & 8.158 & 7.451 & 7.556 \\
\hline $50{ }^{\circ} \mathrm{C}$ & 6.674 & 8.116 & 7.428 & 7.492 \\
\hline $60^{\circ} \mathrm{C}$ & 6.590 & 8.076 & 7.403 & 7.429 \\
\hline $70{ }^{\circ} \mathrm{C}$ & 6.490 & 8.032 & 7.380 & 7.372 \\
\hline$\Delta \delta / \Delta T$ & -8.4 & -4.3 & -2.3 & -6.4 \\
\hline
\end{tabular}


Chemical shift vs Temperature plot for the amide protons in the ${ }^{1}$ H NMR VT studies of Peptide 1b

Chemical shifts of amide protons of peptide $1 \mathrm{~b}$ at various temperatures

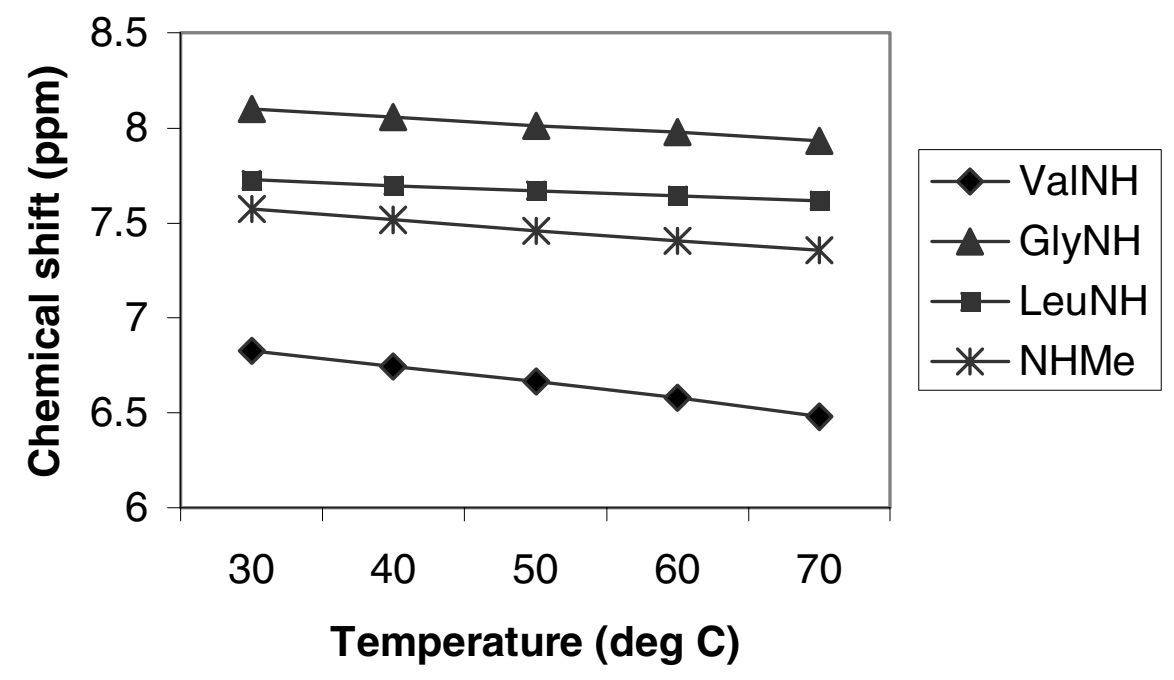

Amide Proton Chemical Shifts obtained during the Variable Temperature Experiment of Peptide 1b

\begin{tabular}{|c|c|c|c|c|}
\hline & Val & Gly & Leu & NHMe \\
\hline $30{ }^{\circ} \mathrm{C}$ & 6.824 & 8.102 & 7.725 & 7.572 \\
\hline $40{ }^{\circ} \mathrm{C}$ & 6.744 & 8.056 & 7.696 & 7.514 \\
\hline $50{ }^{\circ} \mathrm{C}$ & 6.665 & 8.013 & 7.671 & 7.460 \\
\hline $60{ }^{\circ} \mathrm{C}$ & 6.578 & 7.976 & 7.644 & 7.407 \\
\hline $70{ }^{\circ} \mathrm{C}$ & 6.480 & 7.934 & 7.617 & 7.356 \\
\hline$\Delta \delta / \Delta T$ & -8.5 & -4.2 & -2.7 & -5.4 \\
\hline
\end{tabular}




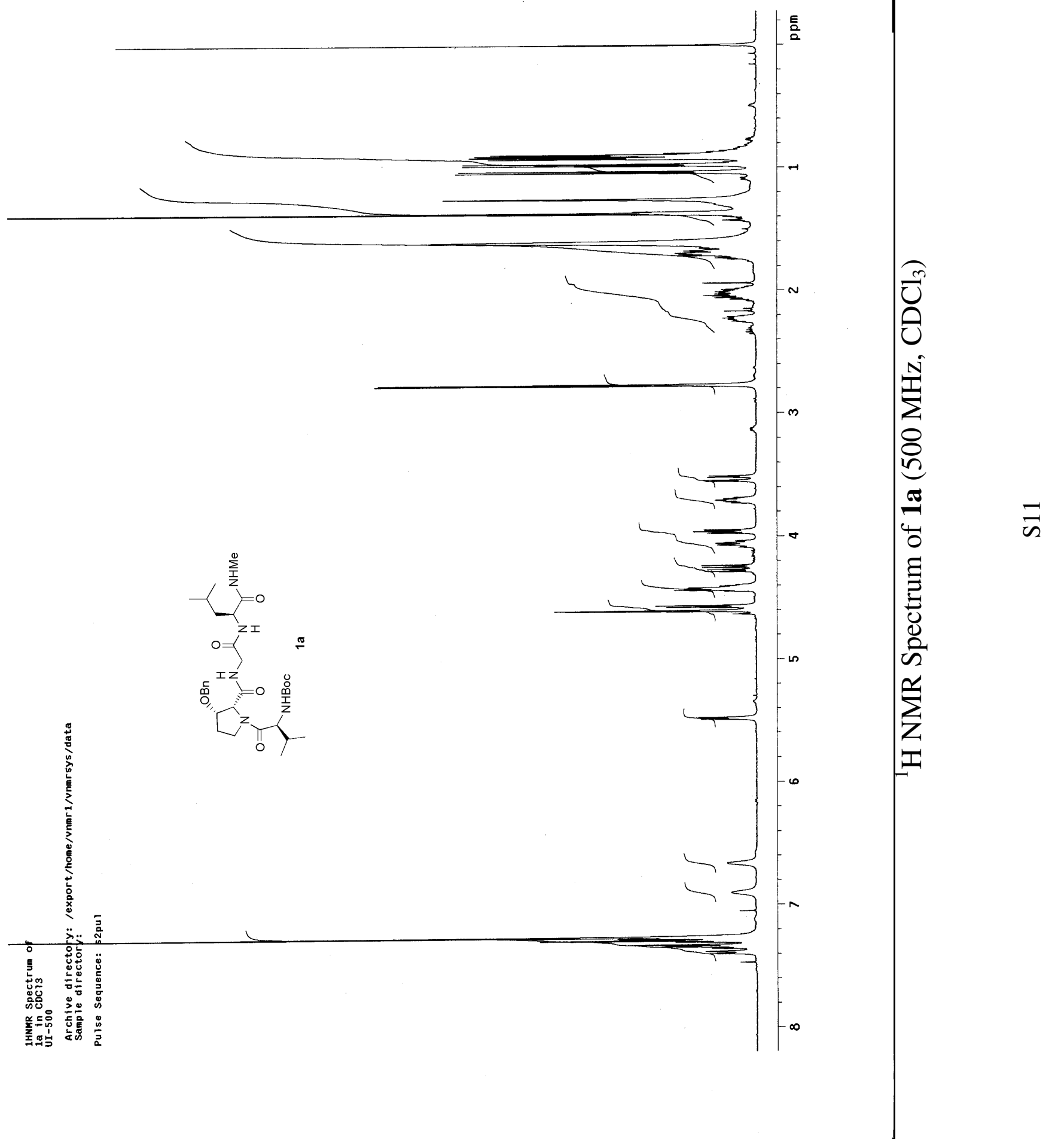




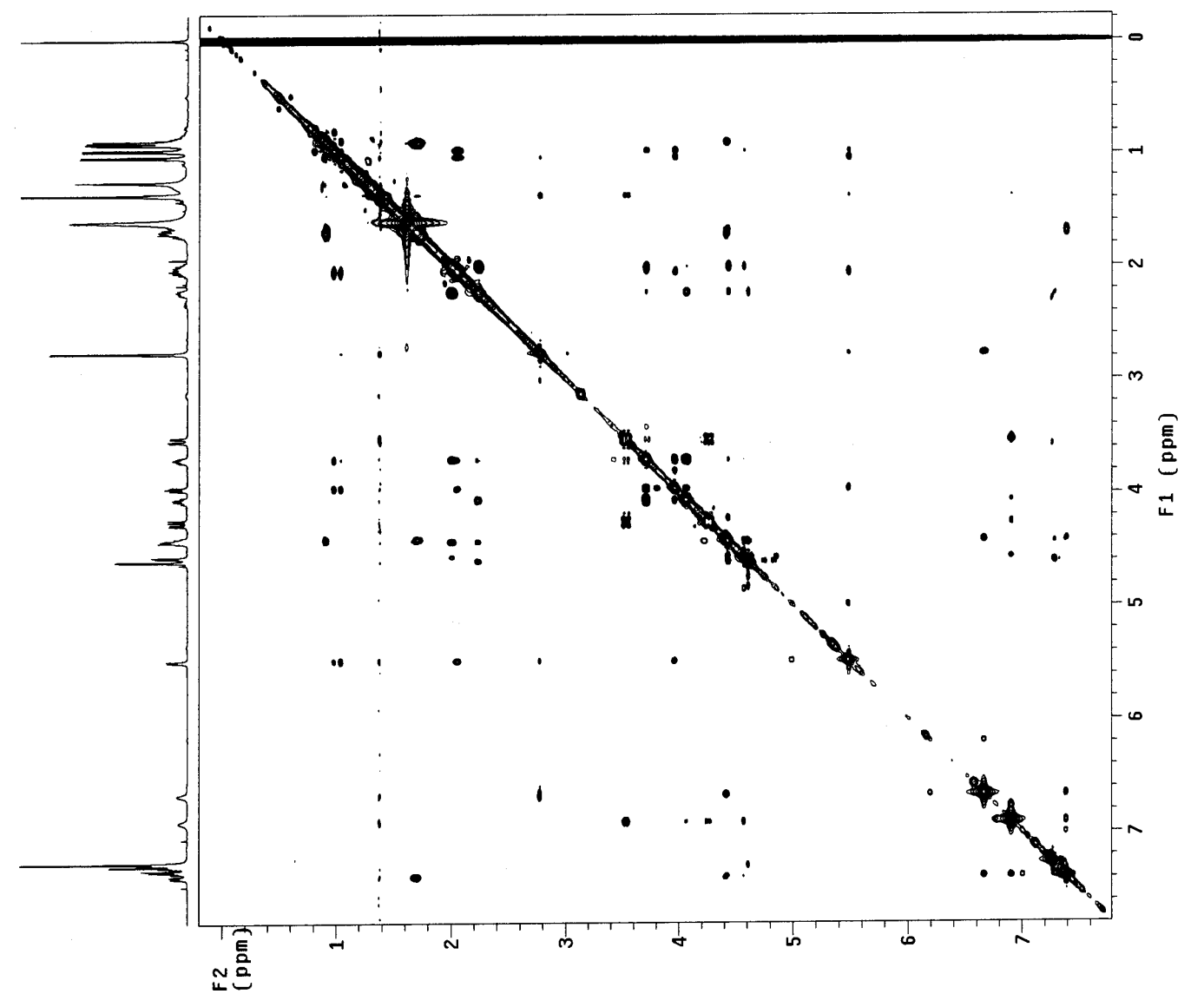

$\frac{N}{n}$
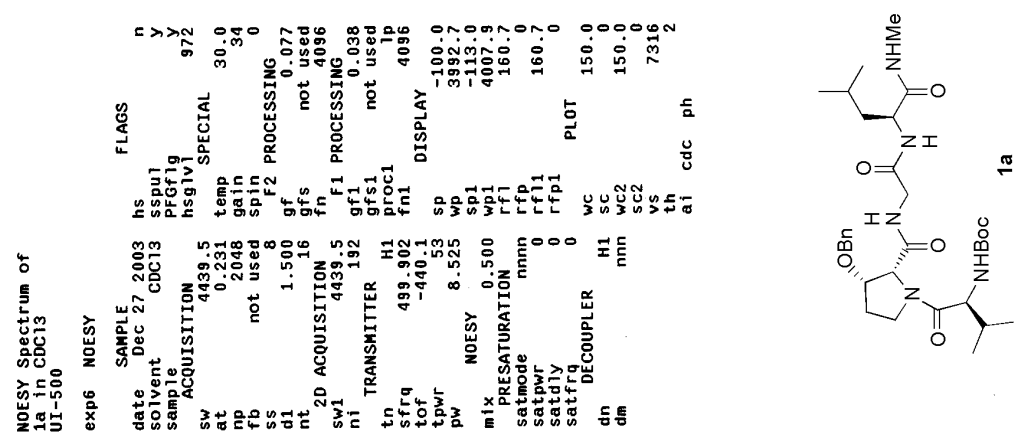


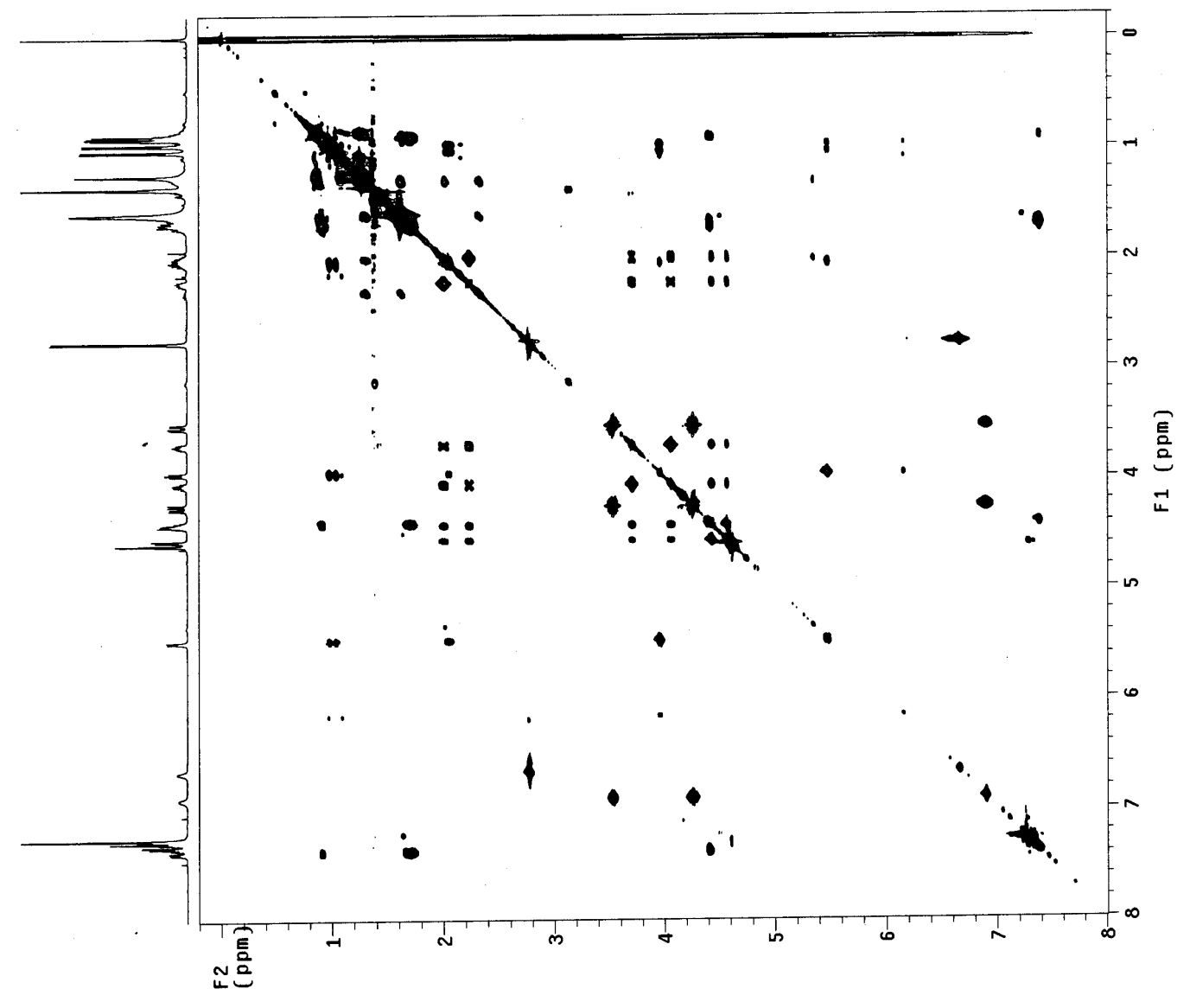

$\frac{m}{n}$
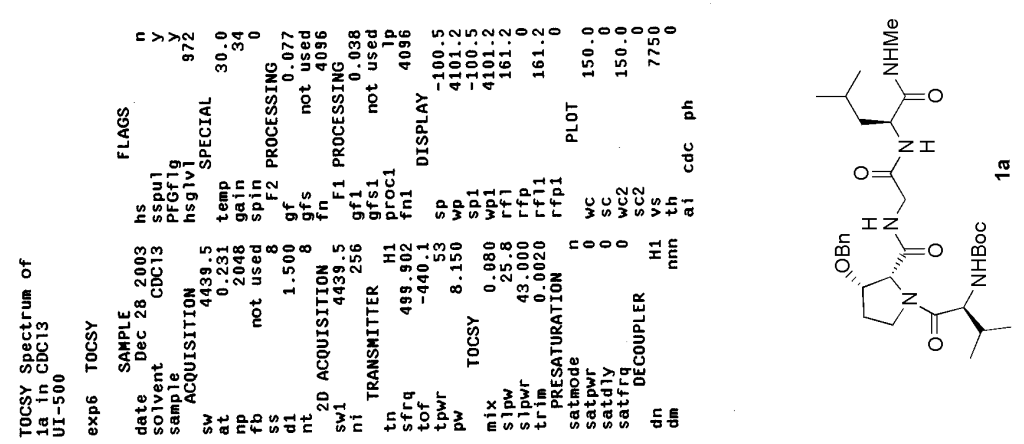


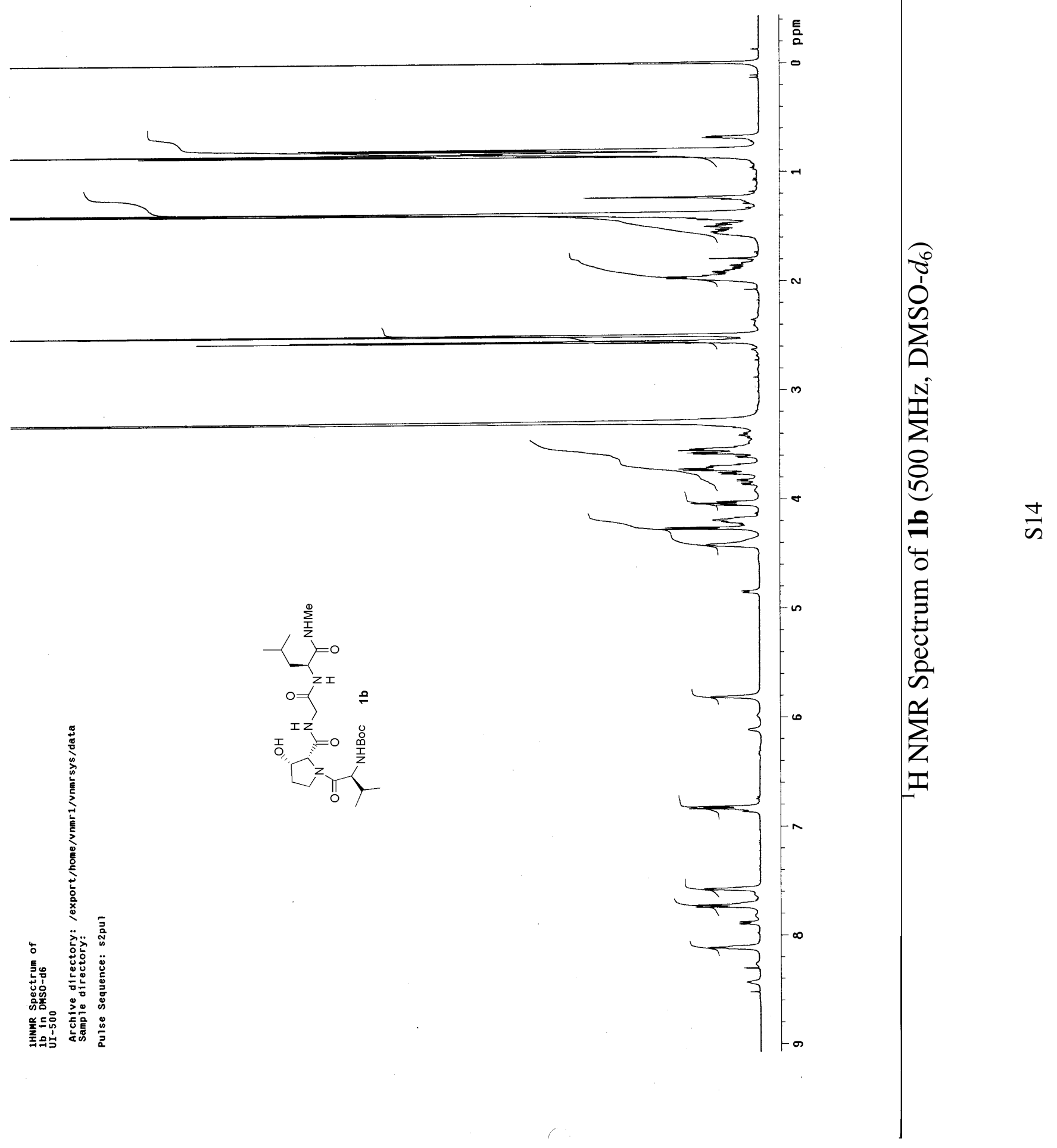




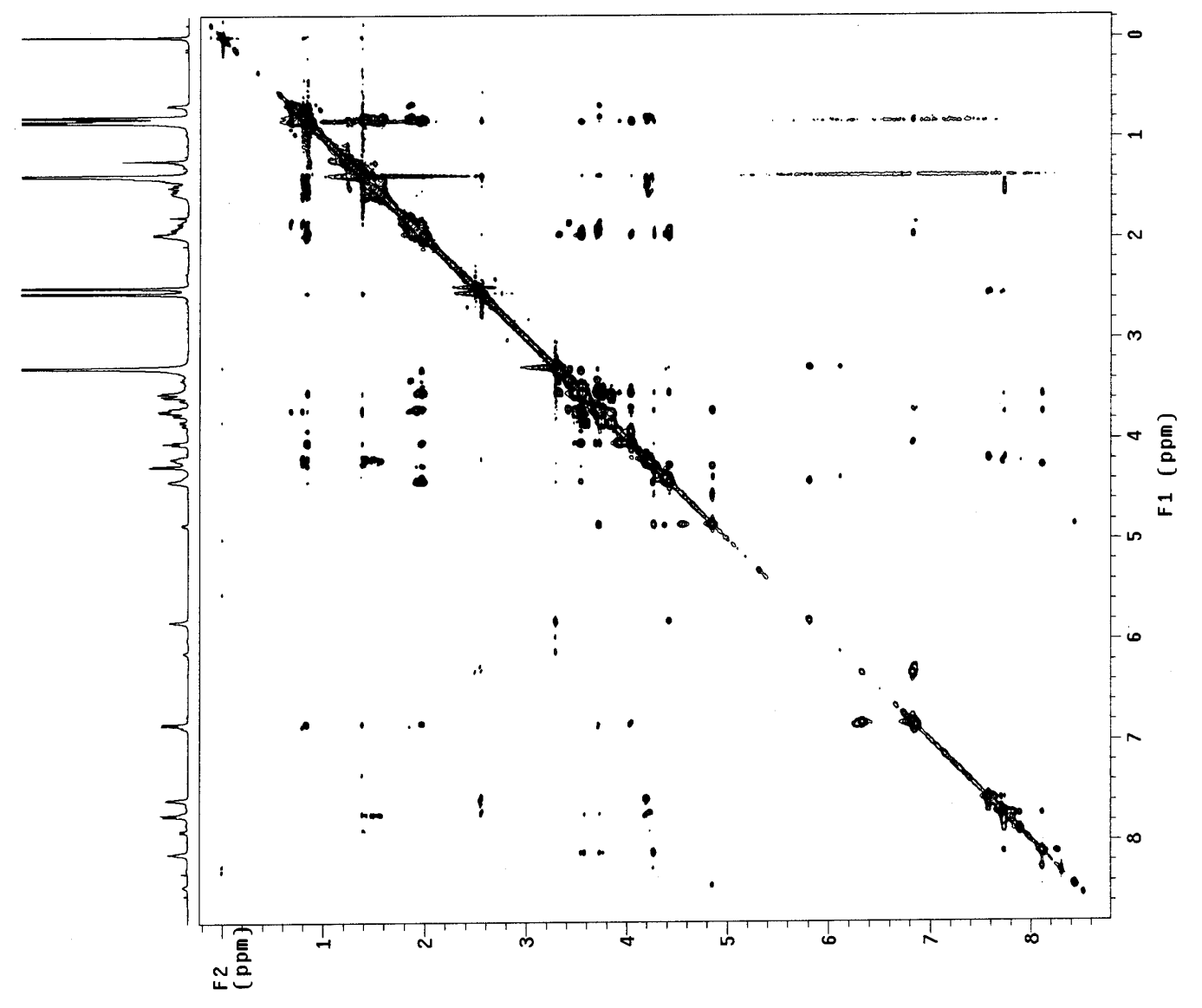

哽

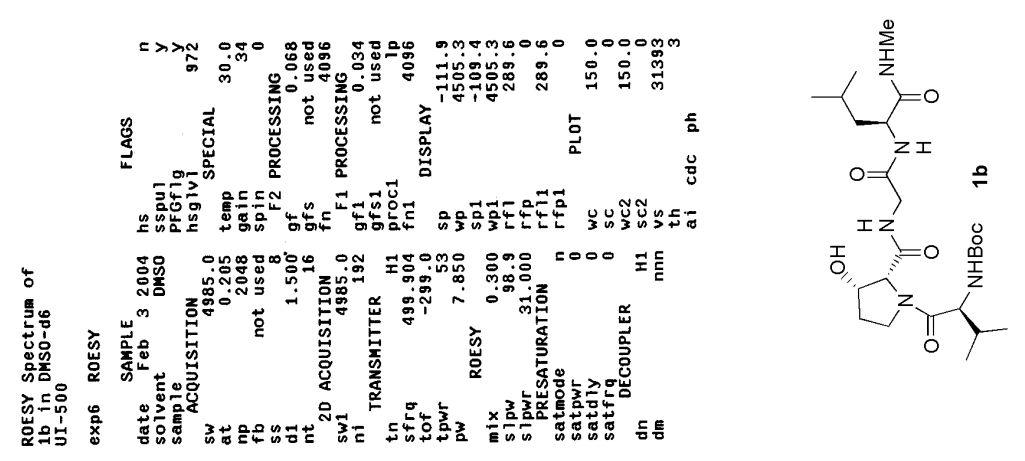



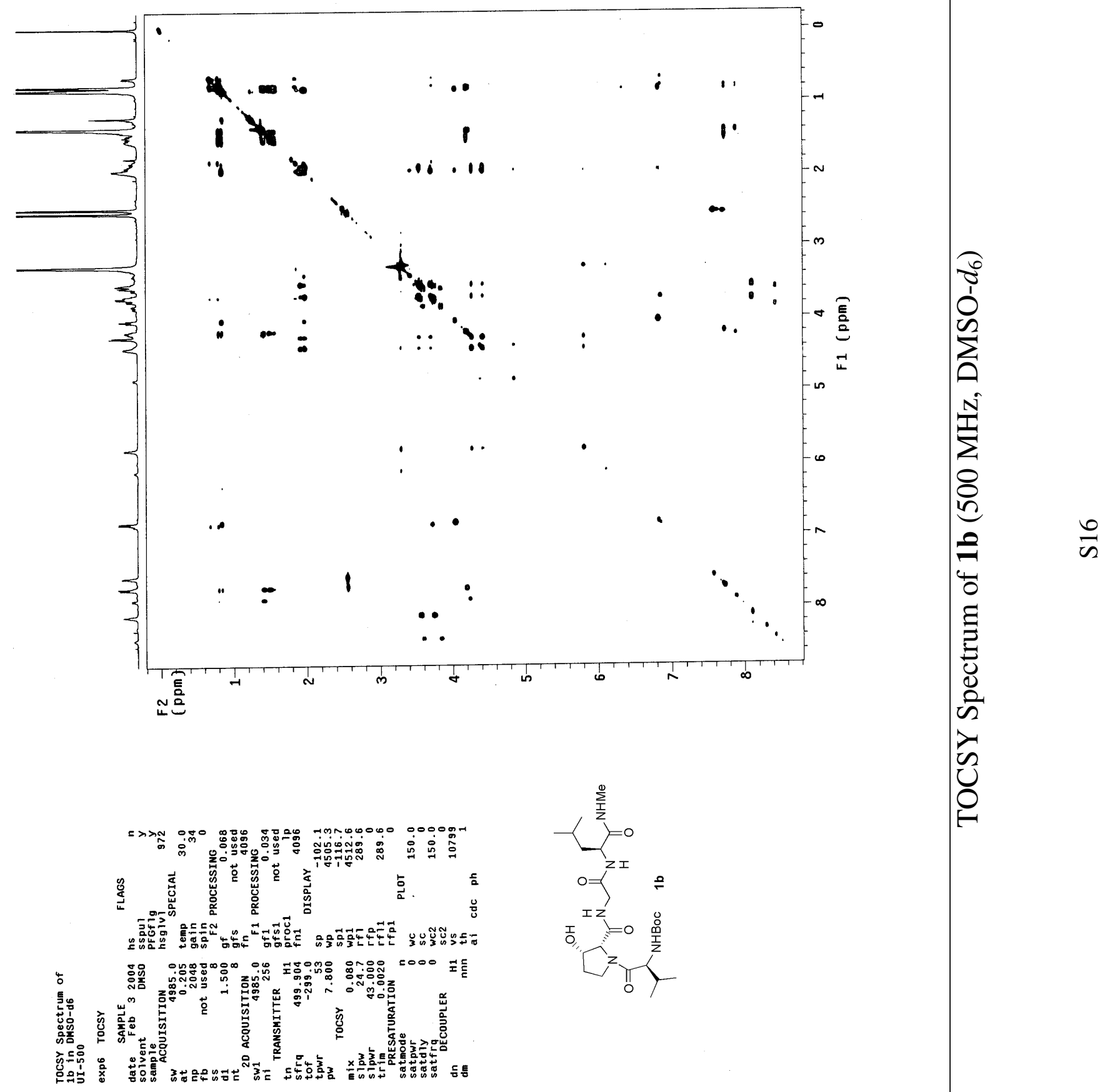


$$
\text { 杉 }
$$




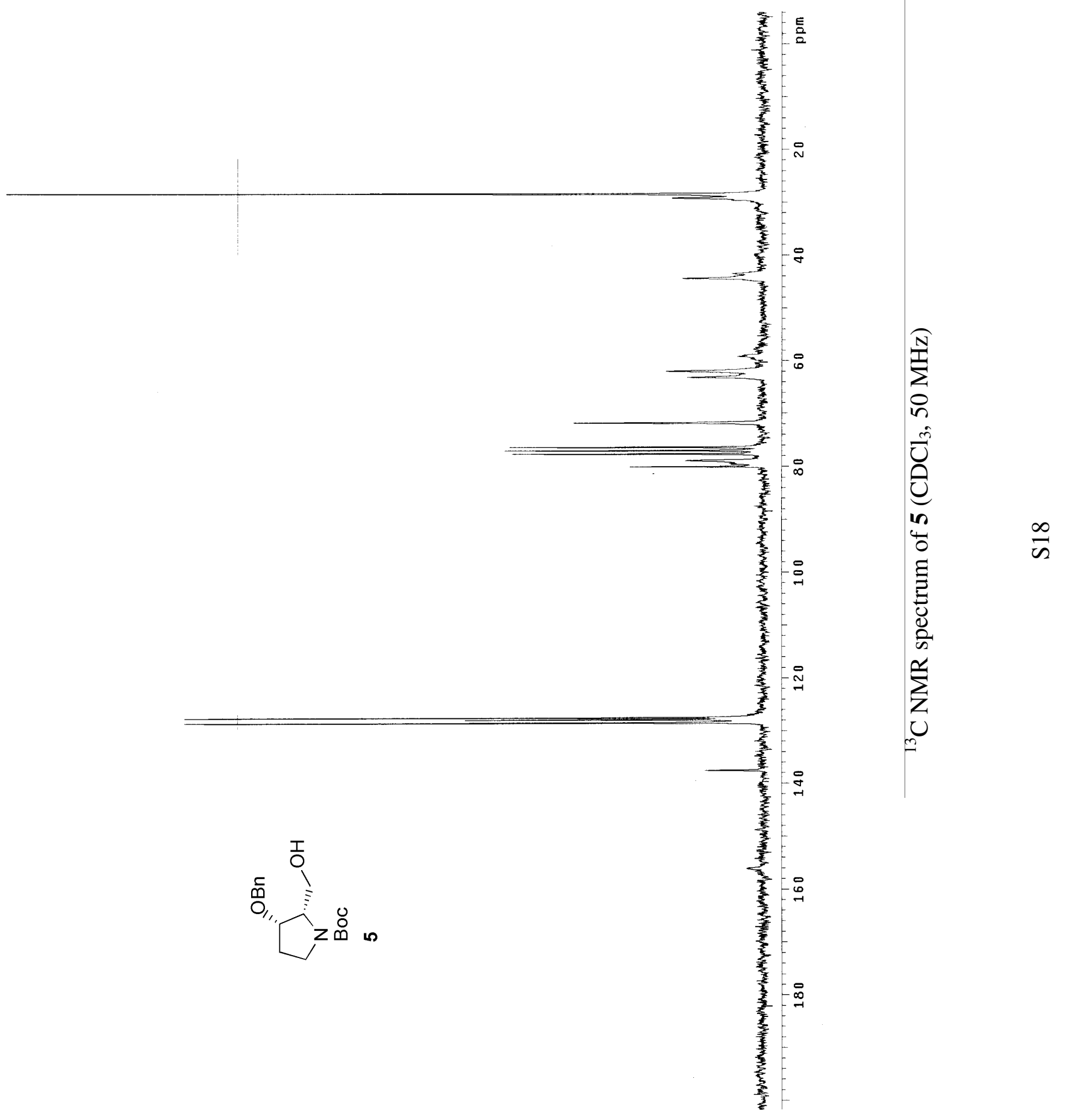




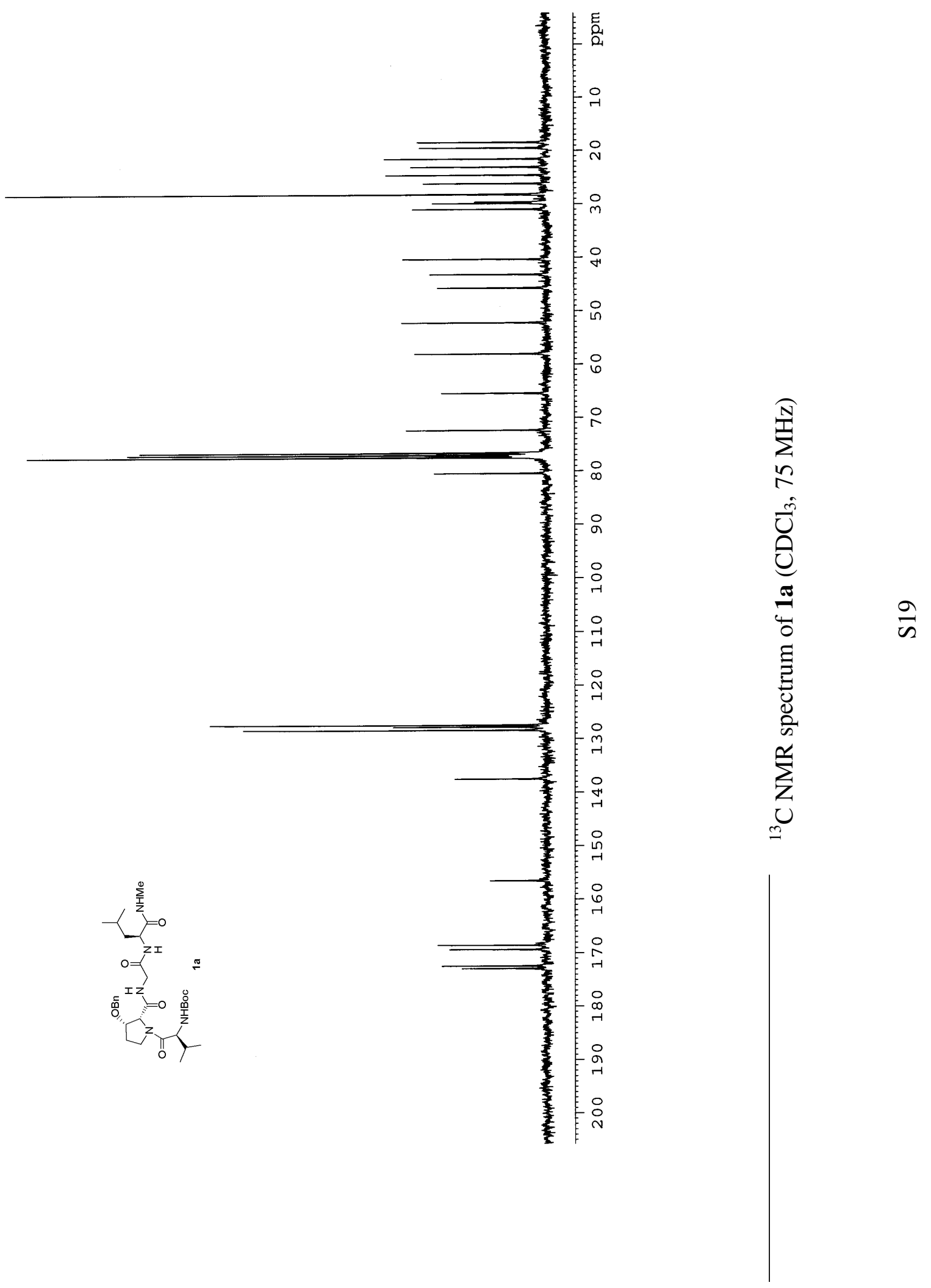




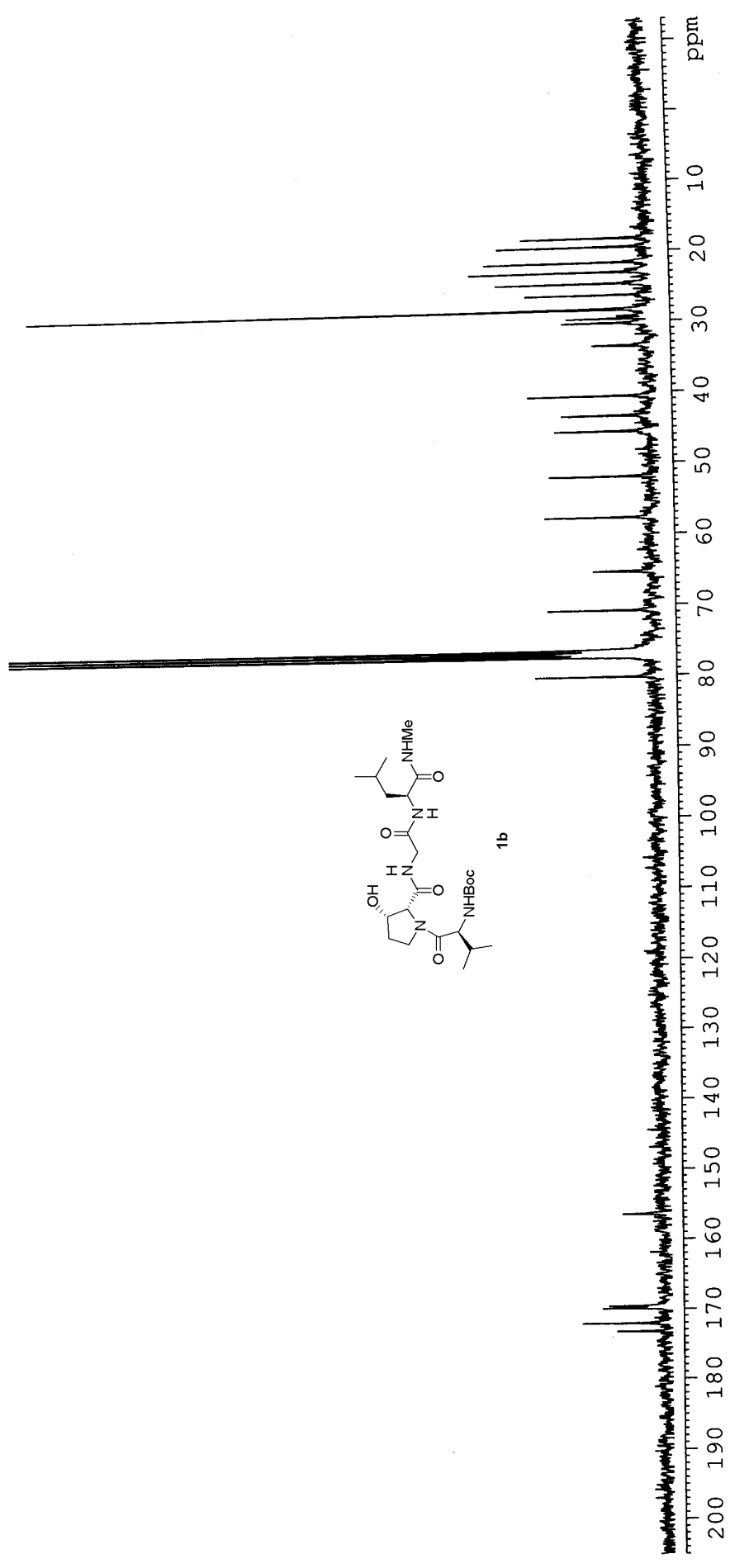

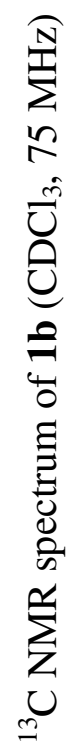

